\title{
Estudio preliminar de los Myxomycetes de Las Yungas de Catamarca (Argentina). I. Ordenes Trichiales y Liceales
}

\author{
Preliminar study of Myxomycetes from Yungas of \\ Catamarca (Argentina). I. OrdersTrichiales and Liceales
}

\author{
Andrea N. Agüero', Adriana I. Hladki²
}

\begin{abstract}
Resumen
Objetivo: Realizar un relevamiento de los órdenes Trichiales y Liceales en Las Yungas de Catamarca. Metodología: Se colectaron muestras frescas y se compararon con colecciones de herbario. Se ilustran las características específicas más representativas. Resultados: Se identificó taxonómicamente a Perichaena chrysosperma, P. depressa, Cribraria aurantiaca, Hemitrichia calyculata, H. serpula, Metatrichia vesparia y Trichia favoginea. Conclusión: Dos especies se registran por primera vez para el Noroeste de la Argentina, se citan dos géneros por primera vez para Catamarca y se amplía el área de distribución de una especie.
\end{abstract}

Palabras clave: Amoebozoa, Argentina, Hongos mucilaginosos, Taxonomía.

\begin{abstract}
Objective: A survey of Trichiales and Liciales orders in the Catamarca's Yungas was performed. Methodology: Fresh samples were collected and compared with herbarium collections. The most representative and specific features are illustrated. Results: Perichaena chrysosperma, $P$. depressa, Cribraria aurantiaca, Hemitrichia calyculata, H. serpula, Metatrichia vesparia and Trichia favoginea, were taxonomically identified. Conclusion: Two species are recorded for the first time to the Northwest of Argentina, two genera are recorded for the first time for Catamarca and the distribution area of a single specie is enlarged.
\end{abstract}

Keywords: Amoebozoa, Argentina, Slime molds, Taxonomy.

\section{Introducción}

Myxomycetes, actualmente considerados dentro de Amoebozoa (Adl et al. 2005; Baldauf 2008), habitan todos los ecosistemas terrestres del planeta y cumplen una importante función al ingerir gran cantidad de bacterias. En cuanto a su diversidad se afirma que la mitad de las especies conocidas se han citado para la región neotropical (Lado y Wrigley de Basanta 2008). Dentro de esta región se encuentra la provincia de Catamarca (Argentina) que cuenta con limitadas investigaciones. A comienzos del siglo XX, Spegazzini (1912) registró en la Quebrada de Pomán y Huillapima la existencia de Arcyria albida Pers.,
A. incarnata (Pers. ex J.F. Gmel.) Pers., A. punicea Pers., Physarum cinereum (Batsch) Pers., y $P$. diderma Rostaf. Posteriormente, Deschamps (1976) en su catálogo sobre los Myxomycetes de la Argentina considera a Arcyria albida un sinónimo de A. cinerea (Bull.) Pers., y a A. punicea de A. denudata (L.) Wetts. Lado et al. $(2009,2011)$ han trabajado en las localidades de Belén y Tinogasta (Catamarca) realizando un destacado aporte a la mixobiota de la provincia y sumando a la misma 28 nuevas especies. Estos estudios se efectuaron en el desierto de monte, donde predominan las cactáceas y suculentas; sin embargo, no se conocen investigaciones en el área que ocupa La Yunga (Figura 1). Moreno et al. (2012)

Cátedra Diversidad Vegetal I, Departamento de Biología, FCEyN-UNCa, Catamarca, Argentina. e-mail: anaguero@yahoo.com

Laboratorio de Micología, Fundación Miguel Lillo, San Miguel de Tucumán, Argentina.

Fecha recepción: Julio 26, 2013 Fecha aprobación: Noviembe 19, 2013 Editor asociado: Torres M. 

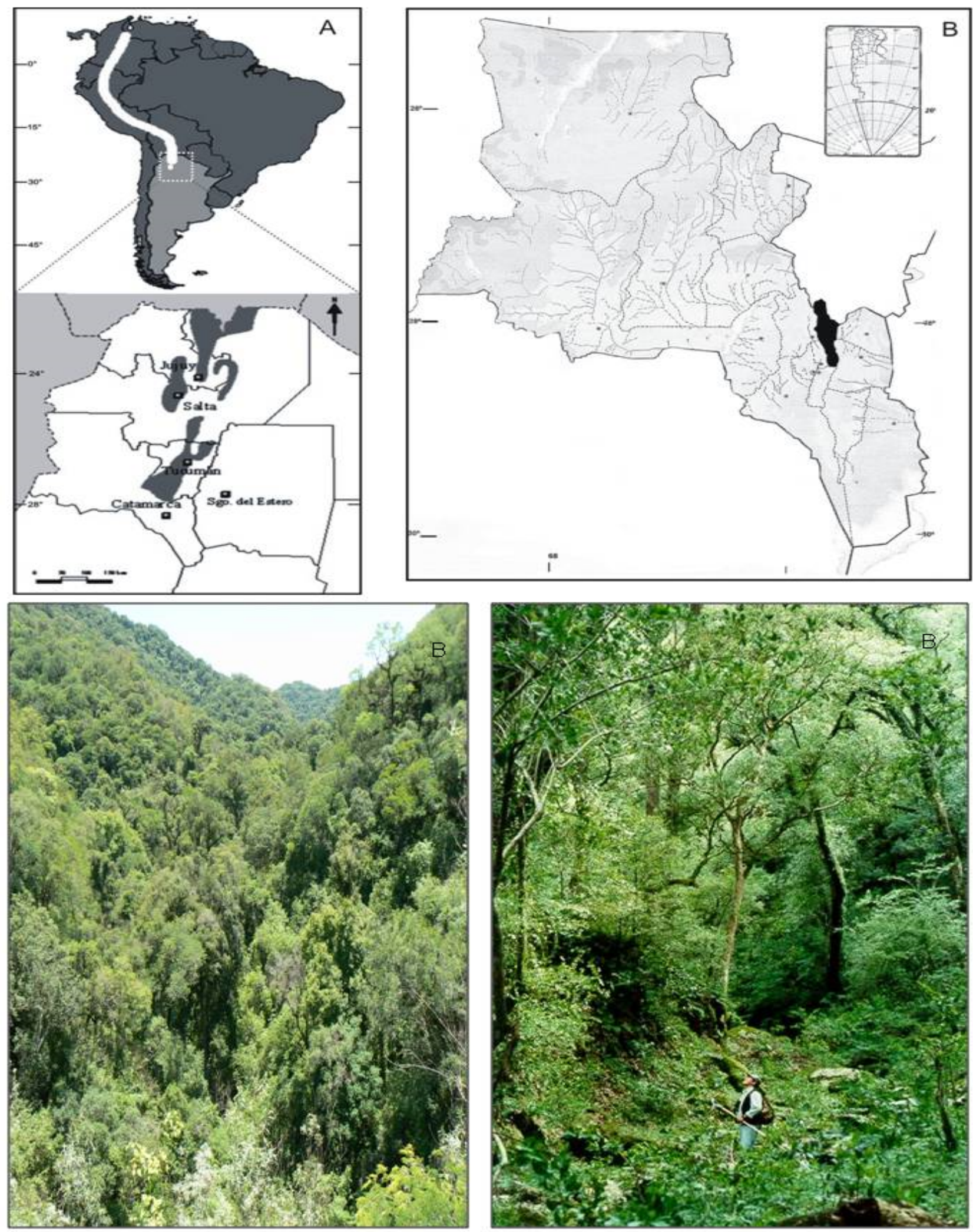

Figura 1. A. Distribución de La Yunga en Sudamérica y en la Argentina. B. Sitio de muestreo en las yungas catamarqueñas. 
desarrollaron otros estudios en la región con especímenes depositados en el Herbario LIL de la Fundación Miguel Lillo, provenientes de La Yunga de Salta, Tucumán y Jujuy, registrando un total de ocho especies.

El interés de la provincia biogeográfica Las Yungas, radica en que, pese a ocupar solo $0,1 \%$ del territorio de Argentina, alberga alrededor del 50\% de su biodiversidad (Brown et al. 1985; Brown y Ramadori 1989; Brown 1995). Esta provincia abarca una superficie estimada de $52.000 \mathrm{~km}^{2}$, extendiéndose desde la frontera con Bolivia $\left(23^{\circ} \mathrm{S}\right)$, Salta, Jujuy y Tucumán, hasta el norte de Catamarca $\left(29^{\circ} \mathrm{S}\right)$, con un gradiente altitudinal de 400 a 3.000 metros (Cabrera 1971).

El fuerte gradiente organiza la vegetación en pisos o franjas fisonómico-florísticas bien característicos, la selva pedemontana (400 a 700 metros de elevación) dominada por la selva de palo blanco y palo amarillo, Calycophyllum multiflorum y Phyllostylon rhamnoides respectivamente, la selva montana (700 a 1500 metros de elevación) un bosque con predominio de especies perennifolias y con estacionalidad hídrica menos marcada que la selva pedemontana, donde se destacan Cinnamomum porphyrium, Nectandra pichurim y Ocotea puberula (Lauraceae), Inga edulis, I. semialata, I. saltensis y Tipuana tipu (Leguminosae), y Blepharocalix salicifolius (Myrtaceae), y el bosque montano (1.500 a 3.000 metros de elevación), lindante con los pastizales de neblina (ubicados altitudinalmente por encima); el paisaje de este piso posee la mayor heterogeneidad estructural, las especies comunes son de clara distribución andina, encontrándose especies de origen austral (Gondwánico), como Podocarpus parlatorei (Podocarpaceae), Roupala meisneri (Proteaceae) y Fuchsia boliviana (Onagraceae) y de origen boreal (Holártico) como Alnus acuminata (Betulaceae), Juglans australis (Juglandaceae), Viburnum seemenii y Sambucus peruviana (Caprifoliaceae), e Ilex argentinum (Aquifoliaceae) (Brown et al. 2002).

Por su amplia distribución latitudinal y su ubicación sobre laderas de montaña de distribución discontinua, Las Yungas en Argentina presentan una sectorización latitudinal (Brown y Ramadori 1989):

a) Sector norte, con los niveles más altos de biodiversidad, en los cordones más occidentales, desde el límite con Bolivia en Salta hasta el sur del PN Calilegua en Jujuy, sector que se reconoce como alta cuenca del río Bermejo o simplemente alto Bermejo;

b) Sector central, con niveles intermedios de biodiversidad, desarrollado sobre los cordones montañosos orientales de Jujuy (Sierras de Santa Bárbara, Centinela y Maíz Gordo), continuando hacia el sur en la provincia de Salta (Serranías del Crestón, Lumbrera, Metán y Candelaria;

c) Sector sur, con los niveles más bajos de biodiversidad, presente en las provincias de Salta, Tucumán y Catamarca.

Por el desconocimiento de la mixobiota de La Yunga, hemos centrado nuestro objetivo de trabajo en la realización de un relevamiento de los órdenes Trichiales y Liceales de Catamarca estudiando morfológicamente colecciones frescas e históricas depositadas en LIL. Ambos órdenes presentan similar coloración de esporas, por lo que Alexopoulos $(1973,1982)$ las consideró relacionadas y posteriores análisis moleculares filogenéticos, realizados por Fiore-Donno et al. (2005), lo corroboran.

\section{Metodología}

Los ejemplares se recolectaron durante exploraciones micológicas realizadas en otoño y primavera en la localidad Cuesta del Totoral, departamento Paclín, Argentina; también se revisaron 30 especímenes depositados en la Mixoteca LIL de la Fundación Miguel Lillo.

Las preparaciones y observaciones microscópicas se realizaron siguiendo la metodología adoptada por Digilio (1950) y Moreno et al. (2012). Para la identificación de los géneros y/o especies se emplearon las claves dicotómicas de Martín y Alexopoulos (1969), Martin et al. (1983) y Lado et al. (2009). Con respecto al tratamiento nomenclatural se sigue a Lado (2005-2013). Las colecciones fueron depositadas en la Mixoteca LIL, con duplicados en la Universidad Nacional de Catamarca. La distribución geográfica de las especies mencionadas está centralizada a Argentina.

\section{Resultados}

1. Cribraria aurantiaca Schrad. Figura 2: A-D Especímenes examinados: ARGENTINA. 


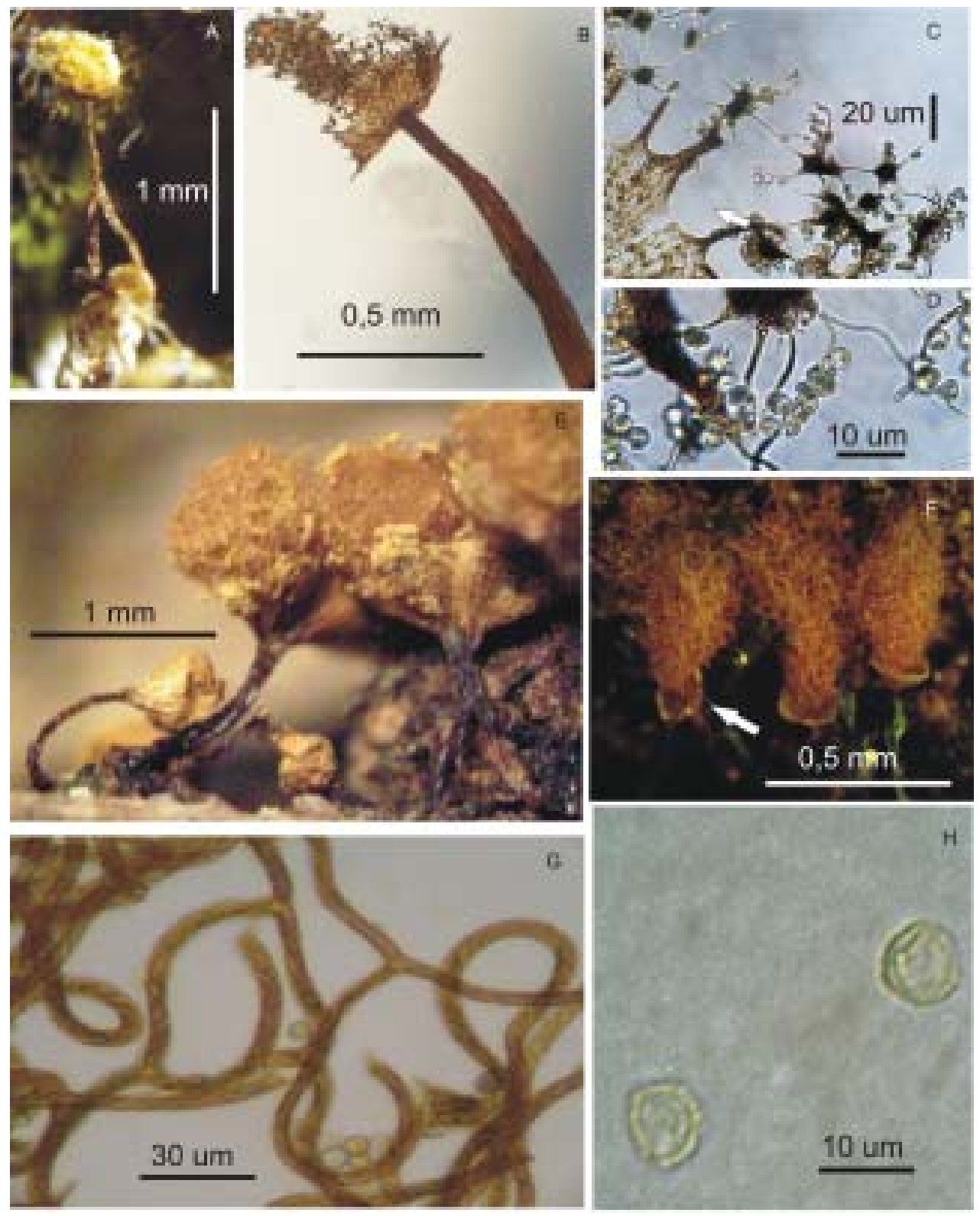

Figura 2. Cribraria aurantiaca Schrad. A. Esporocarpo. B. Esporoteca que muestra su calículo y red peridial. C. Red peridial con nódulos engrosados y gránulos de dictidina. D. Esporas. Hemitrichia calyculata (Speg.) M.L. Farr, E. Esporocarpos agrupados. F. Esporocarpos estipitados con restos de peridio reducido a un calículo basal y abundante capilicio. G. Filamentos del capilicio con bandas espiraladas. H. Esporas.

Catamarca, Paclín, Cuesta del Totoral, Arroyo Los Laureles, $28^{\circ} 6.8^{\prime} 24^{\prime \prime} \mathrm{S}, 65^{\circ} 36^{\prime} 52^{\prime \prime} \mathrm{O}$, a 950 metros de elevación, sobre corteza de dicotiledónea acompañada con musgos, VI-2007, A. Agüero 9, (LIL).

Distribución geográfica: Citada en la provincia de Chubut (Wrigley de Basanta et al. 2010); se cita por primera vez para el norte de Argentina.

Observaciones: El color de la esporoteca, la pre- sencia de nódulos pulvinados y el calículo amplio y bien definido son las características diagnósticas señaladas por Martin y Alexopoulos (1969) para identificar a esta especie. Recientes estudios filogenéticos (Ramírez-Ortega et al. 2009) han demostrado que la existencia de nódulos pulvinados delimita un grupo de especies formado por Cribraria microcarpa, $C$. atrofusca, C. aurantiaca, C. tenella y C. piriformis. 
2. Hemitrichia calyculata (Speg.) M.L. Farr. Figura 2: E-H.

Especímenes examinados: ARGENTINA: Catamarca, Paclín, Cuesta del Totoral, arroyo Los Laureles, $28^{\circ} 6.8^{\prime} 26^{\prime \prime} \mathrm{S}, 65^{\circ} 36^{\prime} 52.8^{\prime \prime} \mathrm{O}$, a 950 metros de elevación, sobre corteza dicotiledónea con musgos, VI-2007, A. Agüero 2, 7, 14 (LIL). Salta, Orán, Urundel, Quebrada del Diablo, ad truncum in silvis, 400 m, 12-VII-1946, Digilio y M. Grassi (LIL 5558). Tucumán, Las Lenguas, ad bambusan sp. in selva montana subtropicali, R. Singer T1282, det G. W. Martin (LIL) (como Hemitrichia stipitata). Tafí Viejo, Taficillo, 4-III-1951, ad limum in selva montana, R. Singer T1337. (LIL) (como Hemitrichia stipitata).

Distribución geográfica: Citada en las provincias de Buenos Aires, Salta y Tucumán (Spegazzini 1880, Deschamps 1976, Crespo y Lugo 2003, Izarduy et al. 2009, Moreno et al. 2012).

Observaciones: Las observaciones sobre algunos de estos materiales fueron presentadas en una contribución anterior (Moreno et al. 2012). Se cita por primera vez el género Hemitrichia para Catamarca y se amplía el área de distribución a Las Yungas argentinas.

3. Hemitrichia serpula (Scop.) Rostaf. ex Lister. Figura 3: A-B.

Especímenes examinados: ARGENTINA: Catamarca, Paclín, Cuesta del Totoral, Arroyo Los Laureles, $28^{\circ} 6.8^{\prime} 22^{\prime \prime} \mathrm{S}, 65^{\circ} 36^{\prime} 50^{\prime \prime} \mathrm{O}$, a 950 metros de elevación, VI-2007, lignícola sobre trozo de rama seca con musgos, A. Agüero 16 (LIL). Tucumán, Tafí Viejo, Sierra de San Javier, Anta Muerta, 900 m., sobre corteza, A.P.L. Digilio y M. Grassi, 12-XI-1946 (LIL 7255).

Distribución geográfica: En la Argentina se la conoce de Buenos Aires, Jujuy y Tucumán (Digilio 1950; Deschamps 1976; Crespo y Lugo 2003; Izarduy et al. 2009; Moreno et al. 2012). Con estos datos se amplía el área de distribución de la especie al noroeste Argentino. Se cita por primera vez para Catamarca, ampliando su área de distribución en las yungas argentinas.

Observaciones: Las observaciones sobre esta especie, fueron expuestas en una contribución anterior (Moreno et al. 2012).

4. Metatrichia vesparia (Batsch) Nann.-Bremek. ex G.W. Martin y Alexop. Figura 3: C-F.

Especímenes examinados: ARGENTINA:
Catamarca, Paclín, Cuesta del Totoral, Arroyo Los Laureles, $28^{\circ} 6.8^{\prime} 24.6^{\prime \prime} \mathrm{S}, 65^{\circ} 36^{\prime} 52.5^{\prime \prime} \mathrm{O}$, a 950 metros de elevación, VI-2007, A. Agüero 8 (LIL). Tucumán, Tafí, San Javier, 15-X-1946, leg. Digilio y Grassi 806 (LIL 7024) (como Hemitrichia vesparium:). Chicligasta, Alpachiri, a 493 metros de elevación, 1-XII-46, A. Garolera (LIL 7552).

Distribución geográfica: Distribuida en Buenos Aires, entre los ríos, Jujuy y Tucumán (Deschamps 1976).

Observaciones: Se cita por primera vez al género Metatrichia para Catamarca y se amplía el área de distribución en la Argentina.

5. Perichaena chrysosperma (Curr.) Lister. Figura 4: A-C.

Especímenes examinados: ARGENTINA: Catamarca, Paclín, Cuesta del Totoral, Arroyo Los Laureles, 28⒍8'26"S, 65'36'52.9"O, a 950 metros de elevación, VI-2007, A. Agüero 15 (LIL), junto con Hemitrichia clavata en corteza de dicotiledóneas con musgos.

Distribución geográfica: Buenos Aires (Deschamps 1976). Se amplía el área de distribución registrándola por primera vez para el noroeste Argentino.

Observaciones: Para la identificación taxonómica se empleó la clave dicotómica aportada por Lado et al. (2009).

6. Perichaena depressa Lib. Figura 4: D-E.

Especímenes examinados: ARGENTINA: Catamarca, Paclín, Cuesta del Totoral, Arroyo Los Laureles, 28⒍8'24.7"S, 65³6'54"O, a 950 metros de elevación, sobre ramita muerta de dicotiledónea, IV-2007, A. Agüero 11 (LIL).

Distribución geográfica: Buenos Aires, Catamarca, Misiones, Salta, San Juan y Tucumán (Deschamps 1976, Lado et al 2011).

Lado et al. (2011) la citan para los departamentos de Belén y Tinogasta (Catamarca) por lo que se amplía el área de distribución en la provincia.

Observaciones: Esta especie presenta los caracteres diagnósticos enunciados por Keller y Eliasson (1992) tales como esporocarpos densamente agregados, aplanados y con filamentos verrugosos, no reticulados y de diámetro variable.

7. Trichia favoginea (Batsch) Pers. Figura 4: F-I

Especímenes examinados: ARGENTINA: 

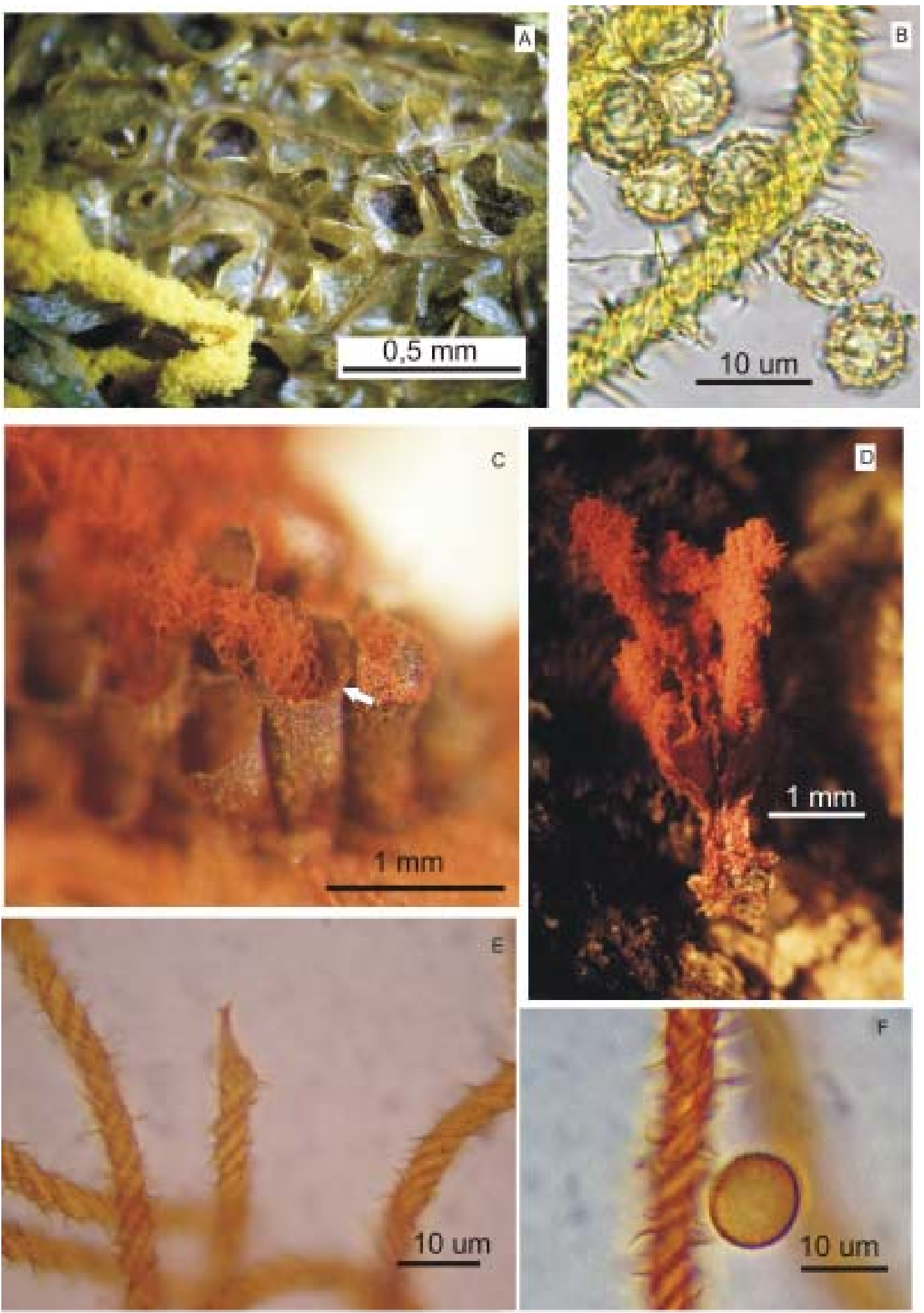

Figura 3. Hemitrichia serpula (Scop.) Rostaf. ex Lister A. Plasmodiocarpo. B. Capilicio y esporas. Metatrichia vesparia (Batsch) Nann.-Bremek. ex G.W. Martin y Alexop. C. Esporocarpos, alguno muestra su dehiscencia circuncisa (flecha). D. Pie común a varios esporocarpos. E. Filamentos del capilicio con espinas y bandas espiraladas. F. Espora y filamento del capilicio. 

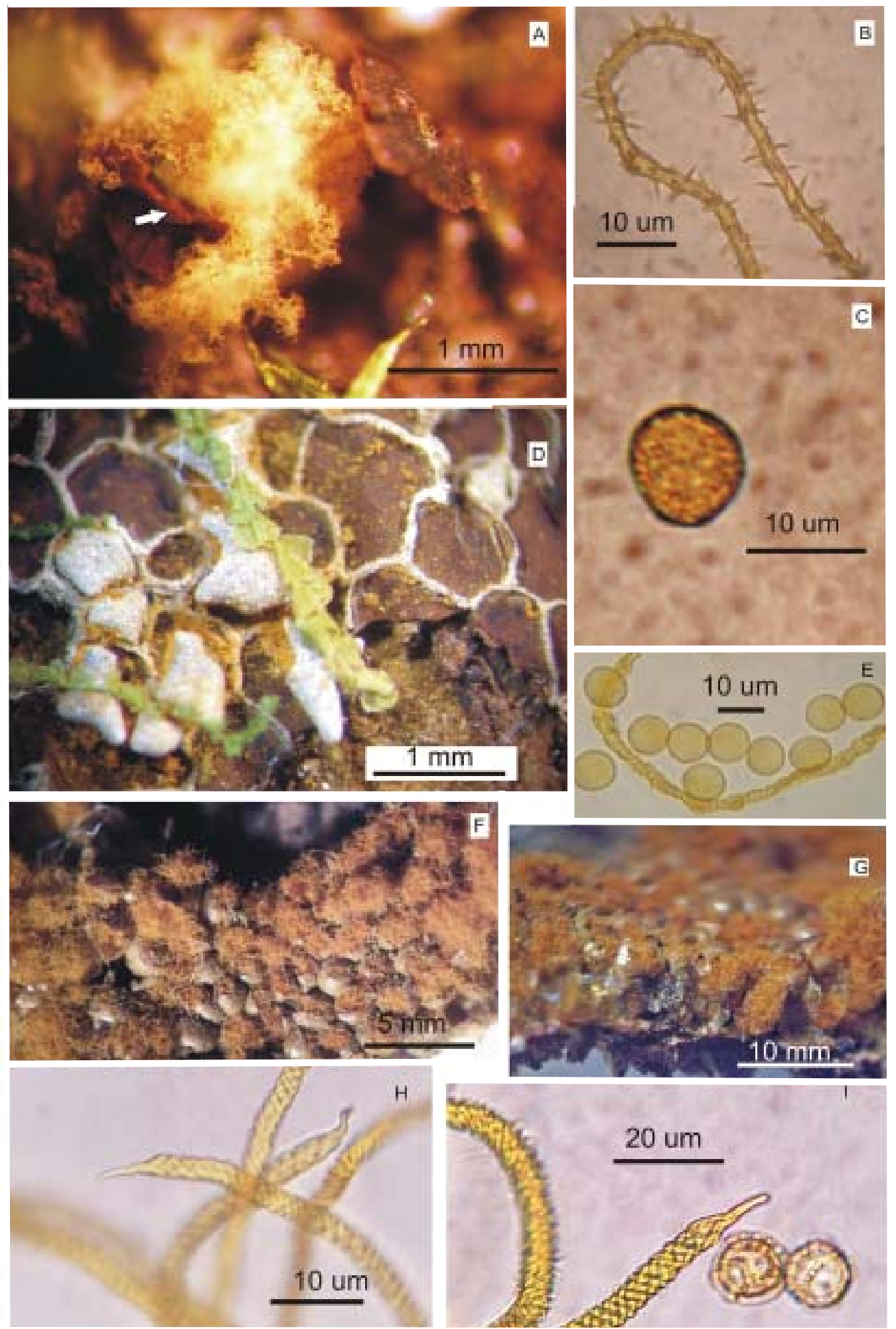

Figura 4. Perichaena chrysosperma (Curr.) Lister. A. Esporocarpo con dehiscencia irregular, B. Filamentos del capilicio con espinas prominentes. C. Esporas finamente verrugosas. Perichaena depressa Lib., D. Esporocarpos, E. Filamento del capilicio y esporas. Trichia favoginea (Batsch) Pers., F. G. Esporocarpos, F. I. Filamentos del capilicio con extremos libres. 
Catamarca, Paclín, Cuesta del Totoral, Arroyo Los Laureles, $28^{\circ} 6.8^{\prime} 23.5^{\prime \prime} \mathrm{S}, 65^{\circ} 36^{\prime} 51^{\prime \prime} \mathrm{O}, 950$ metros de elevación, sobre restos de corteza, VI-2007, A. Agüero 3, 12 (LIL).

Distribución geográfica: Buenos Aires, Chubut, Jujuy, Misiones, Río Negro y Tucumán (Deschamps, 1976, Wringley De Basanta et al. 2010). Se cita por primera vez para Catamarca.

Observaciones: El material presenta notorias coincidencias morfométricas con los especímenes depositados en LIL.

\section{Agradecimientos}

Los autores agradecen a la Lic. M.M. Dios por el apoyo logístico y al Lic. Roberto S. Salinas por el material fotográfico del sector de Yungas referido.

\section{Literatura citada}

Adl SM, Simpson AGB, Farmer MA, et al. 2005. The new higher level classification of eukaryotes with emphasis on the taxonomy of protists. J Eukaryot Mycrobiol. 52 (5): 399-451.

Alexopoulos CJ. 1973. The evolution of the taxonomy of the Myxomyctes. pp. 1-8. In: CV Subramanian (ed.). International Symposium on Taxonomy of Fungi. Part. I. Madras, India.

Alexopoulos CJ. 1982. Morphology, taxonomy and phylogeny. In: Aldrich HC, Daniel HW (eds.). Cell biology of Physarum and Didymium. Vol. 1.New York: Academic Press. pp. 3-23.

Baldauf SL. 2008. An overview of the phylogeny and diversity of eukaryotes. J Syst Evol. 46 (3): 263-73.

Brown AD. 1995. Las selvas de montaña del noroeste de Argentina: problemas ambientales e importancia de su conservación. En: Brown AD, Grau HR (eds.). Investigación, conservación y desarrollo en las selvas subtropicales de montaña. Tucumán: Laboratorio de Investigaciones Ecológicas de las Yungas, UNT. pp. 9-18.

Brown AD, Ramadori ED. 1989. Patrón de distribución, diversidad y caracterización ecológicas de especies arbóreas de las selvas y bosques montanos del noroeste de la Argentina. Anales VI Congreso Forestal Argentino. pp. $177-81$.

Brown AD, Chalukian SC, Malmierca L. 1985. Estudio florístico estructural de un sector de la selva semidecidua del noroeste argentino. I. Composición florística, densidad y diversidad. Darwiniana 26: 27-41.

Brown AD, Grau A, Lomáscolo T, Gasparri NI. 2002. Una estrategia de conservación para las selvas subtropicales de montaña (Yungas) de Argentina. Ecotropicos 15: 14759.
Cabrera AL. 1971. Fitogeografía de la República Argentina. Bol Soc Argent Bot. 14 (1-2): 1-43.

Crespo EM, Lugo M. 2003. Catalogue of Myxomycetes from Argentina. Mycotaxon 87: 91-102.

Deschamps JR. 1976. Los Myxomycetes de la Argentina. Catálogo crítico, distribución y clave de las especies. Physis 35 (90): 147-71.

Digilio APL. 1950. Myxomycetes de Tucumán. Lilloa 23 (4): 365-414.

Fiore-Donno AM, Berney C, Pawlowski J, Baldauf SL. 2005. Higher-order phylogeny of plasmodial slime molds (Myxogastria) based on elongation factor 1-A and small subunit rRNA gene sequences. J Eukaryot Microbiol. 52: $1-10$.

Izarduy CC, Canton NV, Catania M, Hladki AI. 2009. Catálogo de la colección de Myxomycetes depositada en el Herbario Digilio de la Fundación Miguel Lillo. Miscelanea 125: 1-27.

Keller HW, Eliasson UH. 1992. Taxonomic evaluation of Perichaena depressa and Perichaena quadrata based on controlled cultivation, with additional observations on the genus. Mycol Res. 96: 1085-97.

Lado C. 2005-2013. An on line nomenclatural information system of Eumycetozoa. (fecha de acceso: VII-2013). URL disponible en: http://www.nomen.eumycetozoa.com

Lado C, Wrigley de Basanta D. 2008. A review of neotropical Myxomycetes (1828-2008). Anal J Bot. (Madrid) 65 (2): 211-54.

Lado C, Wrigley de Basanta D, Estrada-Torres A, García Carvajal E, Aguilar M, Hernández-Crespo JC. 2009. Description of a new species of Perichaena (Myxomycetes) from arid areas of Argentina. Anal J Bot. (Madrid) 66: 63-70.

Lado C, Wrigley de Basanta D, Estrada-Torres A. 2011. Biodiversity of Myxomycetes from the Monte Desert of Argentina. Anal J Bot. (Madrid) 68 (1): 61-95.

Martin GW, Alexopoulos CJ. 1969. The Myxomycetes. Iowa City: University of Iowa Press.

Martin GW, Alexopoulos CJ, Farr ML. 1983. The genera of Myxomycetes. Iowa City: University of Iowa Press.

Moreno G, Castillo A, Deschamps JR, Hladki AI. 2012. Critical revision of some Myxomycetes kept at the Buenos Aires BAFC Herbarium and Tucuman LIL Herbarium. II. Bol Soc Micol (Madrid) 36: 81-92.

Moreno G, Illana C, Castillo A, García JR. 2001. Myxomycetes de Extremadura. Campiña Sur Postalx, S.L. Iniciativa comunitaria Leader II. pp. 112.

Ramírez Ortega JM, De Luna E, Estrada-Torres A. 2009. A phylogeny of Cribrariaceae among Myxomycetes derived from morphological characters. Mycotaxon. 110: 331-5.

Spegazzini CL. 1880. Fungi Argentini. An Soc Cient Argent. 9 (4): $158-92$.

Spegazzini CL. 1912. Mycetes Argentinenses. An Mus Nac B. Aires 23: 1-2.

Wrigley de Basanta, D, Lado C, Estrada-Torres A, Stephenson SL. 2010. Biodiversity of Myxomycetes in subantartic forests of Patagonia and Tierra del Fuego, Argentina. Nova Hedwigia 90 (1-2): 45-79. 